\title{
High-Throughput and Reliable Isotope Label-free Approach for Profiling 24 Metabolic Enzymes in FVB Mice and Sex Differences ${ }^{\text {[ }}$
}

\author{
Jiamei Chen, Lijun Zhu, Xiaoyan Li, Haihui Zheng, Tongmeng Yan, Cong Xie, Sijing Zeng, Jia Yu, \\ Huangyu Jiang, Linlin Lu, Xiaoxiao Qi, Ying Wang, Ming Hu, and Zhongqiu Liu
}

International Institute for Translational Chinese Medicine, Guangzhou University of Chinese Medicine, Guangzhou, Guangdong, China (J.C., L.Z., X.L., H.Z., S.Z., J.Y., H.J., L.L., X.Q., Y.W., M.H., Z.L.); State Key Laboratory of Quality Research in Chinese Medicine, Macau University of Science and Technology, Macau, China (T.Y.); Department of Pharmacological and Pharmaceutical Sciences, College of Pharmacy, University of Houston, Houston, Texas (M.H.); and Department of Pharmaceutics, School of Pharmaceutical Sciences, Southern Medical University, Guangzhou, Guangdong, China (C.X.)

Received December 14, 2016; accepted March 22, 2017

\begin{abstract}
FVB mice are extensively used in transgenic and pharmacokinetic research. In this study, a validated isotope label-free method was constructed using ultrahigh-performance liquid chromatographytandem mass spectrometry (UHPLC-MS/MS) to quantify 24 drugmetabolizing enzymes (DMEs) in FVB mice. The DMEs include cytochrome P450s (CYP450s/Cyp450s), UDP-glucuronsyltransferases (UGTs/Ugts), and sulfotransferases (SULTs/Sults), which catalyze a variety of reactions to detoxify xenobiotics and endobiotics. The proposed UHPLC-MS/MS method exhibited good range and high sensitivity for signature peptides, as well as acceptable accuracy, precision, and recovery. The protein expression profiles of the DMEs were determined in male and female mice. Overall, the major Cyps,
\end{abstract}

Ugts, and Sults were expressed in male mice followed the rank order: Cyp2c29 > 2e1 > 3a11 > 1a2 > 2d22 > 27a1 > 2c39; Ugt2b5 > 2b1 > 1a6a > 1a9 > 1a1 > 2a3 > 1a2 > 1a5; and Sult1a1 > 3a > 1d1. In contrast, the rank order in female mice was Cyp2c29 > 2e1 > 2c39> $2 d 22>3 a 11>1 a 2>27 a 1$; Ugt1a6a > 2b5 > 1a1 > 2b1 > 2a3 > 1a9> $1 \mathrm{a} 5$ > 1a2; and Sult1a1 > 3a1 > 1d1. Cyp2c29, Cyp1a2, Cyp27a1, Ugt2b1, Ugt2b5 and Ugt2b36 were male predominant, whereas Cyp2c39, Cyp2d22, Cyp7a1, Ugt1a1, Ugt1a5, Sult1a1, Sult3a1, and Sult1d1 were female predominant. This work could serve as a useful reference for the metabolic study of new drugs and for elucidating the effectiveness and toxicity of drugs. The method is stable, simple, and rapid for determining the expression of DMEs in animals.

\section{Introduction}

As the primary organ for metabolism due to high activity of CYPs, UGTs, and SULTs, the liver converts both endogenous and exogenous substances into polar products that are amenable for excretion (Yan et al., 2015). Metabolism and drug-drug interactions (DDIs) involved in metabolism can influence the pharmacokinetics and efficacy/toxicity of drugs (Zhang et al., 2015). Thus, knowledge on metabolism and DDIs would provide useful information to design prodrugs and to predict the outcome of therapy (Margaillan et al., 2015).

Many studies have quantified hepatic DMEs through targeted proteomics (Sakamoto et al., 2011; Fallon et al., 2013a,b). These studies were primarily performed in humans, and only limited data are available for mice. At present, mouse is becoming an increasingly common

This work was supported by the grants of National Natural Science Foundation of China [Grant 81120108025 and 81503466], Science and Technology Project of Guangzhou City [Grant 201509010004], and Guangdong Natural Science Foundation Province [Grant 2015AD030312012].

There is no financial conflict of interests with the authors of this paper. Publication of this paper will not benefit or adversely affect the financial situations of the authors.

J. C. and L.Z. contributed equally to this paper.

https://doi.org/10.1124/dmd.116.074682

SThis article has supplemental material available at dmd.aspetjournals.org. laboratory species because of the high sequence homology between mice and humans and the availability of transgenic and knockout mice (Longo et al., 2011). FVB mice offer many advantages for transgenic research, such as defined inbred background, large litters, and prominent pronuclei (Goelz et al., 1998; Girard et al., 2016). Several studies have used wild-type FVB and efflux transporter knockout mice to investigate the effect of these transporters on drug bioavailability (Zaher et al., 2006; Agarwal et al., 2012; Ge et al., 2015). For this reason, we investigated DMEs in FVB mice in the current study.

It has been proposed that the following DMEs are of clinical relevance in humans: CYP1A2, 2B6, 2C8, 2C9, 2C19, 2D6, 2E1, 3A4, and 3A5; UGT1A1, 1A3, 2B7, and 2B15 (Gröer et al., 2014); SULT1A1, 1A3, 1B1, 1E1, and 2A1 (Riches et al., 2009). Mouse phase I enzymes (Cyp1a2, 1b1, 2c29, 2c39, 2d22, 2e1, 3a11, 7a1, and 27a1) and phase II enzymes (Ugt1a1, 1a5, 1a6a, 1a9, 2b1, 2b5, and Sult1a1) are orthologs of the corresponding human enzymes. Other isoforms, including Cyp3a25, Ugt1a2, 2a3, 2b34, 2b35, 2b36; Sult1d1 and 3a1 are also important in mice. Several epidemiologic and clinical studies have evaluated the effects of sex differences in the pharmacokinetics of drugs. The results have revealed that sex differences in DMEs may influence drug efficacy and safety. Thus, clarifying the contribution of these enzymes, particularly in terms of sex differences, is important for drug efficacy and safety.

DMEs have been studied using bioanalytical methods, such as Western blot (WB), enzyme-linked immunosorbent assay (ELISA),

ABBREVIATIONS: DDI, drug-drug interaction; DME, drug-metabolizing enzyme; LC, liquid chromatography; MLS9, mouse liver S9; MS/MS, tandem mass spectrometry; CYP450, cytochrome P450; QC, quality control; SULT/Sult, sulfotransferase; SPE, solid phase extraction; TFA, trifluoroacetic acid; UGT/Ugt, UDP-glucuronosyltransferase; UHPLC, ultrahigh-performance liquid chromatography; WB, Western blot. 
reverse-transcriptase polymerase chain reaction (RT-PCR), and enzyme activity assays (Ohno and Nakajin, 2009; Joo et al., 2014; Shi et al., 2015). However, these methods intended to measure the abundance of DMEs have many limitations. For example, WB requires specific antibodies that are rarely available because of highly homologous enzymes within the DMEs. The application of RT-PCR to detect mRNA is unreliable because the mRNA expression level may not always correlate with protein level. Enzymatic activities are widely used for indirectly reflecting the protein amounts. However, a variety of DMEs always have broad and often overlapping substrates specificities. Furthermore, these methods are usually semiquantitative and low throughput. Thus, development of an accurate and high-throughput method for determining DME protein levels is highly desirable. An alternative approach to accurately quantify DMEs using liquid chromatography tandem mass spectrometry (LC-MS/MS) is broadly used (Seibert et al., 2009). The present new approach is able to sensitively (detection of fmol protein) and easily (quantification of multiple proteins) determine the absolute amounts of proteins with a high degree of sequence homology (distinction of molecular weight less than $1 \mathrm{Da}$ ) in complex biologic matrices, such as quantifying CYP and UGT superfamilies in biologic tissues or cultured cell lines (Schaefer et al., 2012; Achour et al., 2014). MS-based protein quantification approaches, which are characterized by selective, precise, and large dynamic range, are highlighted as a promising method for basic research on drug development such as DDI studies and pharmacokinetic and pharmacodynamic modeling.

In this study, we successfully developed an accurate and high throughput method to systematically quantify 24 DMEs in male and female FVB mice. The developed method and animal model can provide deep insights into the hepatic disposition and metabolism of drugs. Understanding sex differences may clarify the molecular basis for differences in drug disposition between males and females.

\section{Materials and Methods}

Chemicals and Reagents. Ammonium bicarbonate, dithiothreitol, iodoacetamide, trifluoroacetic acid (TFA), formic acid, acetic acid, and phenylmethanesulfonyl fluoride were purchased from Sigma-Aldrich Co. (St. Louis, MO). Sequencing grade modified trypsin was provided by Promega (Madison, WI). All peptides and internal standard (purity $>95 \%$ ) were purchased from All peptides were confirmed again and all peptides were purchased from APeptide Co., Ltd. (Shanghai, China). Solid phase extraction (SPE) cartridges (C18 $50 \mathrm{mg}, 3 \mathrm{ml}$ ) were obtained from J.T. Baker (Philipsburg, NJ). Coomassie brilliant blue for protein measurement was purchased from Bio-Rad (Hercules, CA).

Instrumentation and Mass Spectrometry. An Agilent 1290 series UHPLC system and an Agilent 6490 triple quadrupole mass spectrometer interfaced with an electrospray ionization source (Agilent Technologies, Santa Clara, CA) was applied for quantifying all samples.

A Poroshell C18 column $(2.1 \mathrm{~mm} \times 100 \mathrm{~mm}, 2.7 \mu \mathrm{m}$; Agilent Technologies $)$ with an injection volume of $10 \mu \mathrm{l}$ was used for separation. The sample rack temperature was maintained at $10^{\circ} \mathrm{C}$, and the analytical column temperature was set at $40^{\circ} \mathrm{C}$. The mobile phase A was $0.1 \%$ formic acid in water and the mobile phase B was $100 \%$ acetonitrile. To achieve chromatographic separation, a linear gradient was set at a flow rate of $0.3 \mathrm{ml} / \mathrm{min}$ as follows: $0-1.8$ minutes, $5-8 \% \mathrm{~B}$; $1.8-2$ minutes, $8-10 \% \mathrm{~B}$; 2-3.5 minutes, $10-14 \% \mathrm{~B} ; 3.5-10$ minutes, $14-35 \% \mathrm{~B}$; $10-12$ minutes, $35-80 \%$ B; $12-14$ minutes, $80-80 \%$ B ; $14-15$ minutes, $80-5 \%$ B and post time was set to 2 minutes for column equilibration.

The mass spectrometer was operated in positive ion mode to monitor the $\mathrm{m} / \mathrm{z}$ transitions for all peptides. The detailed information of peptides was listed in Supplemental Table 1. For all peptides, at least three transitions of each peptide were selected for quantification. To gain high sensitivity, one of the most abundant fragments was used for quantification, whereas at least two of the fragments were used for qualitative analysis for high selectivity. To optimize the collision energies, a scheduled multiple reaction monitoring acquisition method was developed. The following main mass working parameters were set as follows: capillary voltage $3 \mathrm{kV}$; nozzle voltage $1500 \mathrm{~V}$; gas temperature $200^{\circ} \mathrm{C}$; sheath gas temperature $250^{\circ} \mathrm{C}$; sheath gas flow of $11 \mathrm{l} / \mathrm{min}$; gas flow of $14 \mathrm{l} / \mathrm{min}$. Data were collected and analyzed using the Mass Hunter software (version B.06.00, Agilent Technologies).

Animals. Male and female FVB mice (11 weeks) were obtained from Vital River Laboratory Animal Technology Co. Ltd (Beijing, China). The animals were housed in a controlled condition, with ambient temperature of $24-26^{\circ} \mathrm{C}$ and humidity of 50-60\% and a 12-h light/dark cycle. All animal experiments were approved by the Institutional Animal Care and Use Committee of the Guangzhou University of Chinese Medicine. Before the experiment, the animals were fasted overnight with free access to water.

Preparation of Mouse Liver S9 Fractions. S9 fractions from livers were isolated from male and female FVB mice. MLS9 were prepared as previously described with minor modifications (Zhu et al., 2010; Tang et al., 2012). Mouse livers were washed, perfused with solution $\mathrm{B}\left(8 \mathrm{mM} \mathrm{KH}_{2} \mathrm{PO}_{4}, 5.6 \mathrm{mM} \mathrm{Na}_{2} \mathrm{HPO}_{4}\right.$, $1.5 \mathrm{mM}$ EDTA), and then minced. The minced livers were homogenized using a motorized homogenizer in an ice-cold homogenization buffer $(50 \mathrm{mM}$ potassium phosphate, $250 \mathrm{mM}$ sucrose, $1 \mathrm{mM}$ EDTA, pH 7.4) and then centrifuged at 12,000 $g$ for 15 minutes at $4^{\circ} \mathrm{C}$. The fat layer and the pellet were discarded, and the supernatant was collected and stored at $-80^{\circ} \mathrm{C}$ until use. Protein concentrations (typically $5-30 \mathrm{mg} / \mathrm{ml}$ ) of MLS9 were measured by Coomassie brilliant blue, and bovine serum albumin was used as the standard.

Sample Preparation and Tryptic Digestion. S9 protein $(120 \mu \mathrm{g})$ from MLS9 was digested as previously described with minor modifications (Sridar et al., 2013). Samples were denatured and reduced at $95^{\circ} \mathrm{C}$ for 10 minutes in ammonium bicarbonate digestion buffer $(50 \mathrm{mM}, 90 \mu \mathrm{l})$, which was spiked with dithiothreitol in $5 \mathrm{mM}$ final concentration. After cooling down, $10 \mu \mathrm{l}$ iodoacetamide ( $10 \mathrm{mM}$ final concentration) was added for alkylation at least 30 minutes in the dark at room temperature. Subsequently, the mixture (final volume, $124 \mu \mathrm{l}$ ) was incubated with trypsin at $37^{\circ} \mathrm{C}$ for 4 hours (trypsin:protein $=1: 50$ ). The digested reaction was terminated with TFA $(120 \mu \mathrm{l}, 0.5 \% \mathrm{v} / \mathrm{v})$, and $20 \mu \mathrm{l}$ of internal standard $(200 \mathrm{nM})$ was added. After centrifugation at $12,000 \mathrm{~g}$ for 10 minutes, the supernatants were dried under nitrogen. One milliliter of TFA: acetonitrile $(0.1: 100, \mathrm{v} / \mathrm{v})$ and $1 \mathrm{ml}$ of TFA: water $(0.1: 100, \mathrm{v} / \mathrm{v})$ were used to prepare SPE for sample cleaning, respectively. The samples were loaded into the conditioned SPE and then washed with $1 \mathrm{ml}$ of $0.1 \%$ TFA. Finally, the analytes were eluted with $1 \mathrm{ml}$ of acetonitrile-water-TFA mixture (60:40:0.1, v/v/v). After the eluent was evaporated to dryness under nitrogen and the samples were reconstituted with $200 \mu 1$ methanol-water-acetic acid mixture (5:95:0.1, v/v/v) and then centrifuged for 30 minutes at $18,000 \mathrm{~g}$. The supernatant was determined by UHPLC-MS/MS.

Method Validation. According to the U.S. Food and Drug Administration guidelines, the fundamental parameters, including linearity, accuracy, precision, matrix effect, recovery, and stability should be evaluated in the present work.

To prepare the calibration curves and quality control (QC) samples, digested Insect Cell Control Supersomes (BD Biosciences, Woburn, MA). were used as a blank matrix and was spiked with various of each peptide to obtain the following concentrations for calibration values: $0.39,0.78,1.56,3.13,6.25,12.5,25,50$, 100 , and $200 \mathrm{nM}$ and QC samples were prepared in 1.56 or 3.13, 12.5, and $100 \mathrm{nmol}$ or $12.5,25$, and $100 \mathrm{nmol}$ concentrations. The calibration curves were prepared in the same way as described in the sample preparation section.

The precision and accuracy of the method were evaluated by measuring the standard samples at three concentration levels $(12.5,25$, and $100 \mathrm{nM}$ for Cyp1a2, Cyp1b1, Cyp2d22, Cyp3a25, Cyp7a1, Cyp27a1, Ugt1a1, and Ugt1a2; 1.56, 12.5, and $100 \mathrm{nM}$ for other signature peptides of DMEs). Intra-day precision was determined using six replicates QC samples in the same day. Inter-day precision was measured using 18 replicates QC samples for 3 days. According to each calibration curve, the concentration of target protein can be calculated. Matrix effects were investigated by spiking target peptide standards into trypsinized MLS9.

The extraction recovery was calculated by comparing the concentrations obtained from analytes before the extraction with those from samples to which analytes were added after the extraction. Autosampler stability was investigated by measuring sample extracts immediately after preparation and after storing them in a cooled autosampler $\left(10^{\circ} \mathrm{C}\right)$ for 3 and 7 days.

Data Analysis. Independent sample $t$ test was conducted using SPSS 17.0 to evaluate statistical differences. $P<0.05$ denotes statistical significance.

\section{Results}

Calibration Curves. To date, it has been hard to find validation data for quantification of DMEs that meet the requirements of current bioanalytical 
guidelines. Therefore, the present study was to develop and validate modified method. To avoid producing major interference, we used control insect Supersomes instead of mammalian liver S9 as the matrices to construct calibration curves. The developed method was selective for determining all proteospecific peptides in digested insect Supersome and in digested MLS9. In most cases, peptide concentration linearly correlated with the analytical signal among the overall validation range $(1.56-200 \mathrm{nM})$. The correlation coefficients $\left(r^{2}\right)$ for all targeted signature peptides were better than 0.980 (1/x weighting) (Supplemental Table 2). Multiple reaction monitoring chromatograms of the standards are shown in Fig. 1.

Accuracy and Precision. The accuracy and precision data are shown in Supplemental Table 3. The inaccuracy values of the majority of signature peptides were less than $25 \%$ at three tested concentration levels, except for Ugt1a1 ( $-28.6 \%, 43.1 \%$, and $-10.2 \%$ at low, middle, high concentration). Intra- and inter-day imprecision were below $15 \%$ in samples at all three concentration levels.

Matrix Effect. It is common to use isotope-labeled peptides to overcome the matrix effects for protein quantification. However, the standard addition method is an alternative for determining the matrix

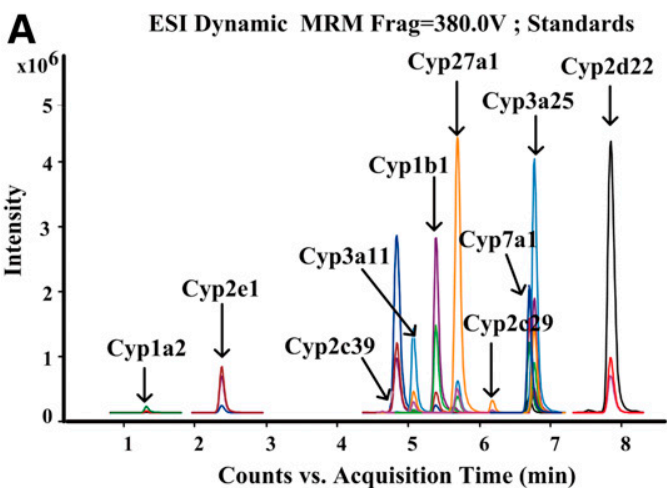

B

ESI Dynamic MRM Frag=380.0V ; Standards
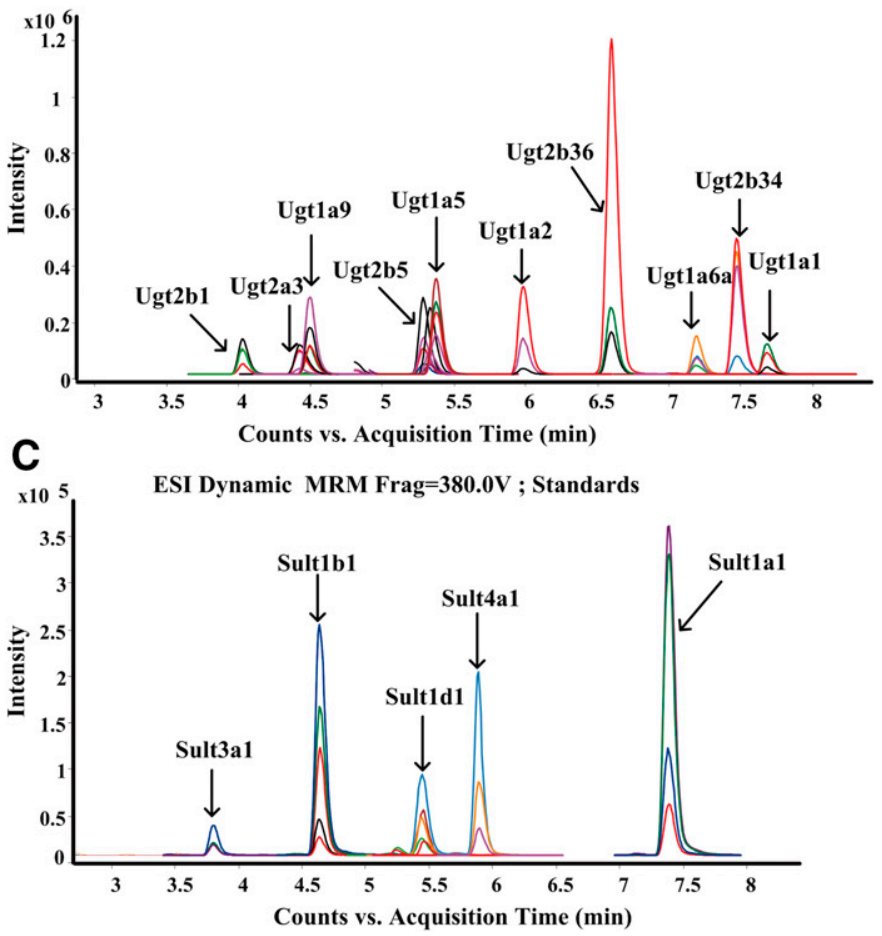

Fig. 1. Dynamic multiple reaction monitoring chromatograms for the developed LC-MS/MS methods applied to measure proteotypic peptides. (A), (B), and (C) represent the standards of Cyps, Ugts, and Sults, respectively. effects. In the present work, eight samples were randomly selected as test samples. A known amount of external standard peptides was spiked into test samples, and the samples were evaluated by UHPLC-MS/MS. The added amount of the peptides was calculated by subtracting the amount of peptides originally contained in the MLS9 from that in the spiked samples. The matrix effect was assessed by comparing the calculated amounts of spiked peptides with the known (i.e., externally added) amounts. The inaccuracy values of most measured peptides were less than 20\%; however, the inaccuracy values of Cyp2c39, Ugt1a6a, Ugt2b34, and Sult1a1 in one of eight samples were higher than $20 \%$ $(24 \%, 26.9 \%, 25.2 \%$, and $-32.2 \%$, respectively). Nevertheless, the accuracies of our determinations were within acceptable ranges, suggesting that the standard addition method could be applied to measure matrix effects.

Extraction Recovery and Stability. The recovery was calculated due to all samples were processed using SPE. As shown in Supplemental Table 5, the recovery of most signature peptides was higher than $80 \%$ at the three concentration levels. The extraction efficiency for Sult2a1 and Sult4a1 was slightly lower but within the acceptable range of $70-80 \%$. The recovery values were within acceptable ranges, suggesting that this SPE protocol could be applied to clean-up protein digestion. The analytes displayed varying properties for all signature peptides. Therefore, it was hard to obtain the ideal range of $70-130 \%$. Autosampler stability was measured for 3 and 7 days at $10^{\circ} \mathrm{C}$. As shown in Supplemental Table 5, all peptides demonstrated sufficient stability ( $\pm 20 \%$ of the initial concentrations at low, medium, and high concentration) for 3 days in the autosampler rack. However, acceptable stability was observed for the middle and high conditions. By contrast, at low concentrations, isoforms Ugt2a3, 2b1, 2b5, and Sult1d1 failed to meet the requirements of the current bioanalytical guidelines (greater than $25 \%$ ) for 7 days.

Enzyme Expression Profiles of 24 DMEs in S9 Fractions Prepared from Male FVB Mice. As shown in Fig. 2A-4A, all isoforms of the 24 DMEs could be quantified in the same individual samples. Fig. 2B and Supplemental Table 6 show that the most abundant Cyp was Cyp2c29, with an average protein concentration of $88.2 \mathrm{pmol} / \mathrm{mg}$, followed by Cyp2e1, at $34.1 \mathrm{pmol} / \mathrm{mg}$. The average protein concentrations of Cyp3a11, Cyp1a2, and Cyp2d22 were within a similar range at $15.9,15.6$, and $11.9 \mathrm{pmol} / \mathrm{mg}$, respectively. The average protein concentrations of five other measurable Cyps were below $5 \mathrm{pmol} / \mathrm{mg}$. Fig. 3B and Supplemental Table 6 show that among the Ugts in the liver, Ugt2b5, Ugt2b1, and Ugt1a6a consistently had the highest enzyme expression levels, with average protein concentrations of 38.6, 27.8, and $25.7 \mathrm{pmol} / \mathrm{mg}$. Moderate levels of Ugt2b36, Ugt1a9, Ugt1a1, Ugt2a3, and Ugt2b34 were detected in the liver, at 9.6, 9.5, 8.9, 8.6, and $8.2 \mathrm{pmol} / \mathrm{mg}$ proteins, respectively. Ugt2b35, Ugt1a2, and Ugt1a5 were readily detectable, but their expression levels were generally lower (below $5 \mathrm{pmol} / \mathrm{mg}$ proteins) compared with other Ugt enzymes. The protein expression levels of three Sults isoforms were shown in Fig. 4B and Supplemental Table 6. Sult1a1 was the most abundant enzyme in the liver, at $8.2 \mathrm{pmol} / \mathrm{mg}$ protein, accounting for approximately half of the total Sults content, followed by Sult $3 \mathrm{a} 1$ ( $5.3 \mathrm{pmol} / \mathrm{mg}$ protein), and Sult1d1 was $3.5 \mathrm{pmol} / \mathrm{mg}$ protein. To estimate the percentage contributions of individual DME isoforms to the total enzymes present in the liver, the mean expression levels for each enzyme were calculated and expressed as the percentage of sum of all the means of all Cyps or Ugts or Sults present in the sample. The complete data were plotted in Fig. 2C4C. Several recent studies have addressed the absolute concentration of CYP or UGT isoforms in human liver microsomes. The ranks of DMEs in the current study were in good agreement with the published data in humans, suggesting that the FVB mouse may be an ideal animal model for predicting drug kinetics and toxicity in humans. 
A

Cyp1a2

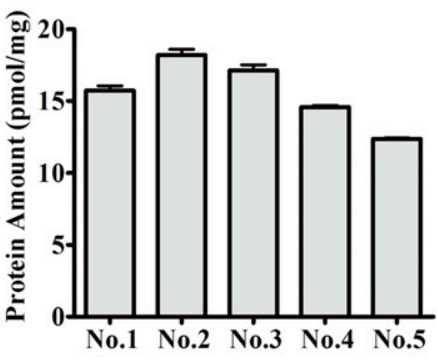

Сур2c39

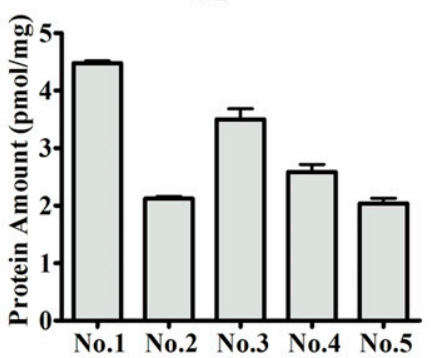

Cyp3a11

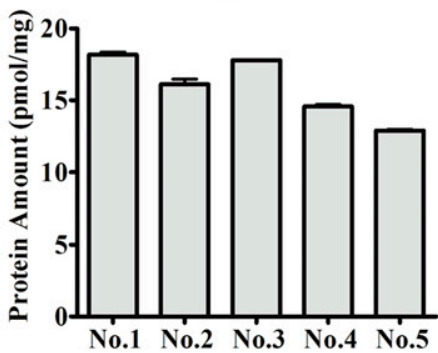

Cyp27a1

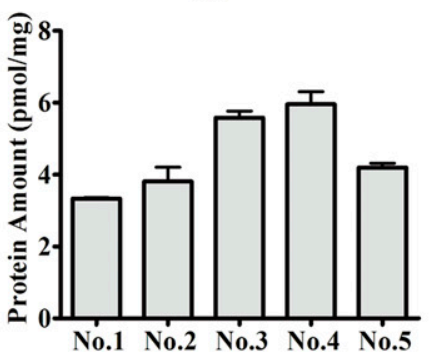

B

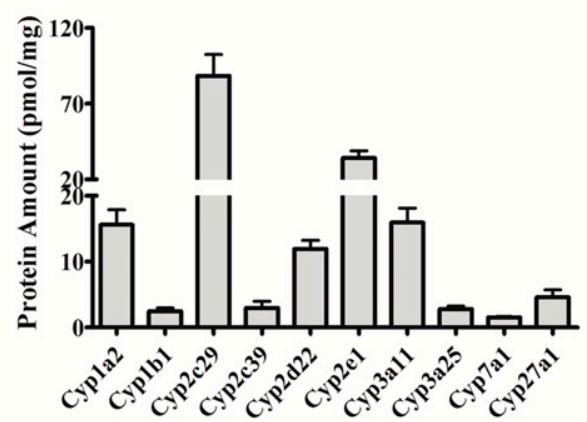

Cyp1b1

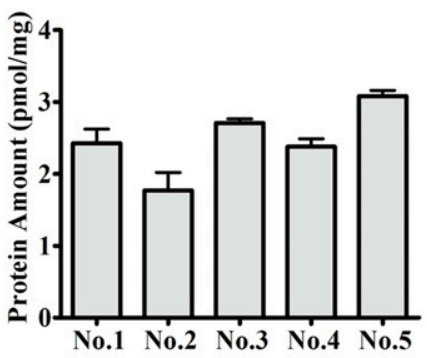

Cyp2d22
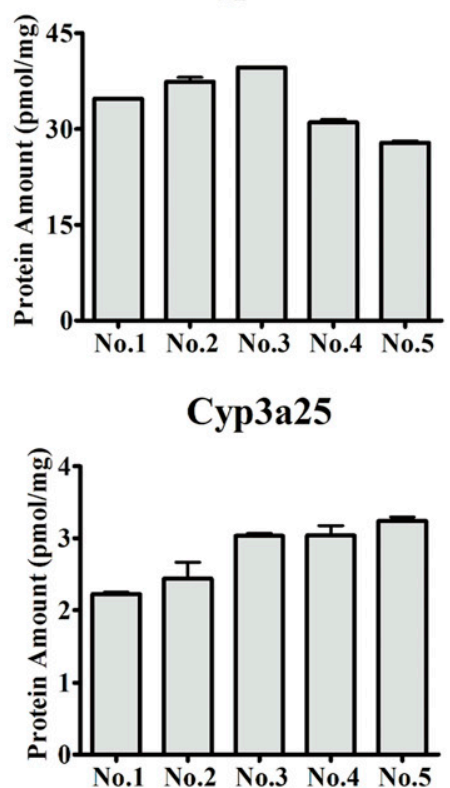

Cyp2c29

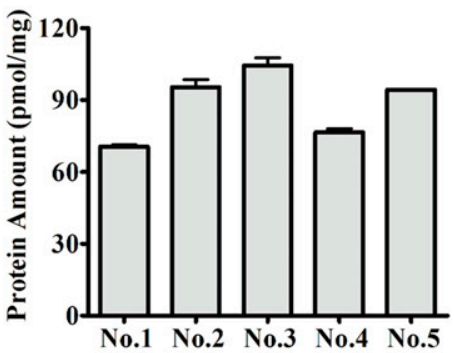

Cyp2e1
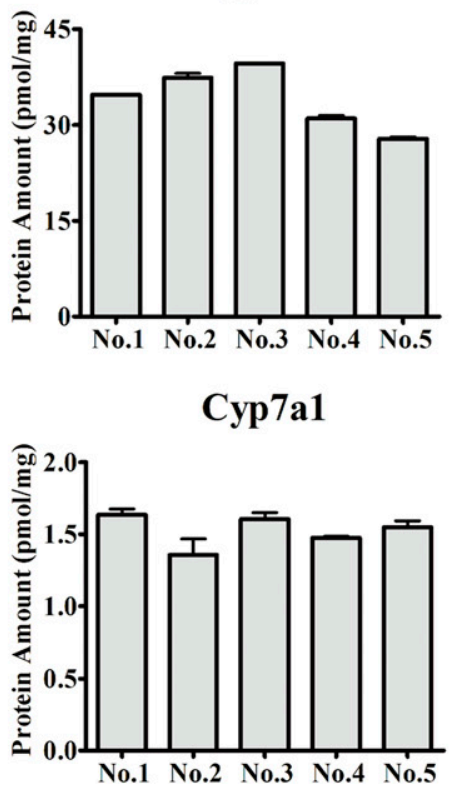

C

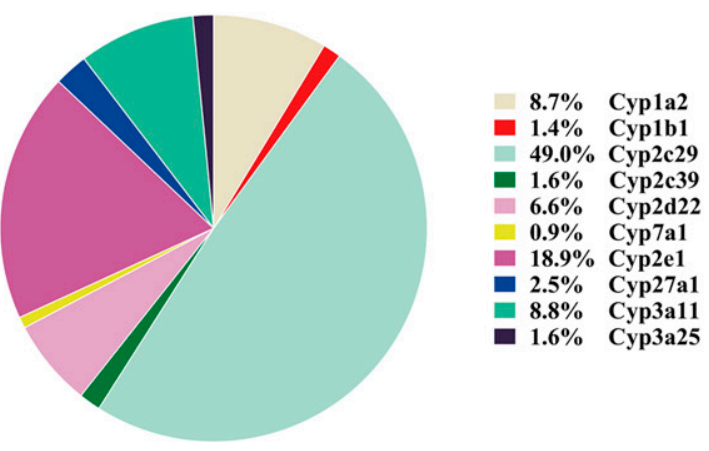

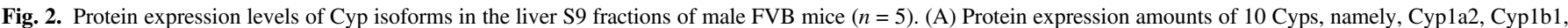

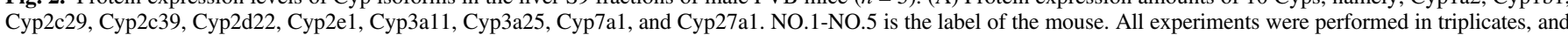

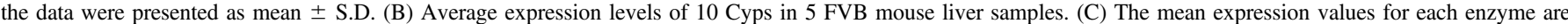
displayed as percentages of the total sum of the quantified 10 Cyps present in the liver. 

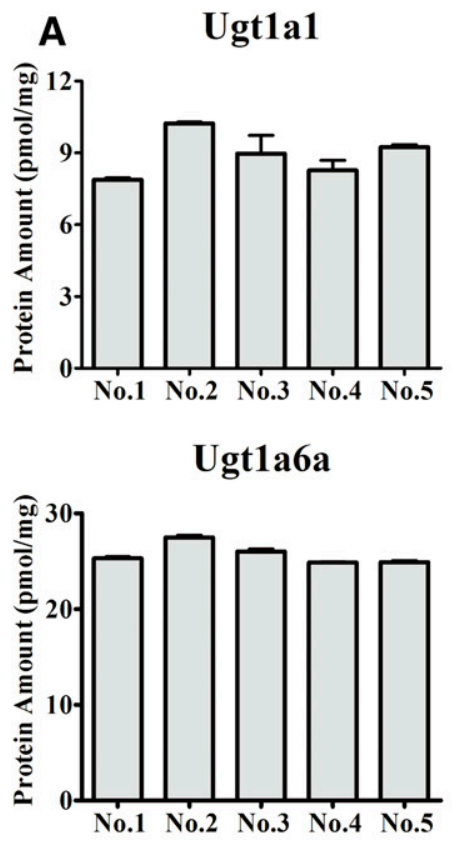

Ugt2b1
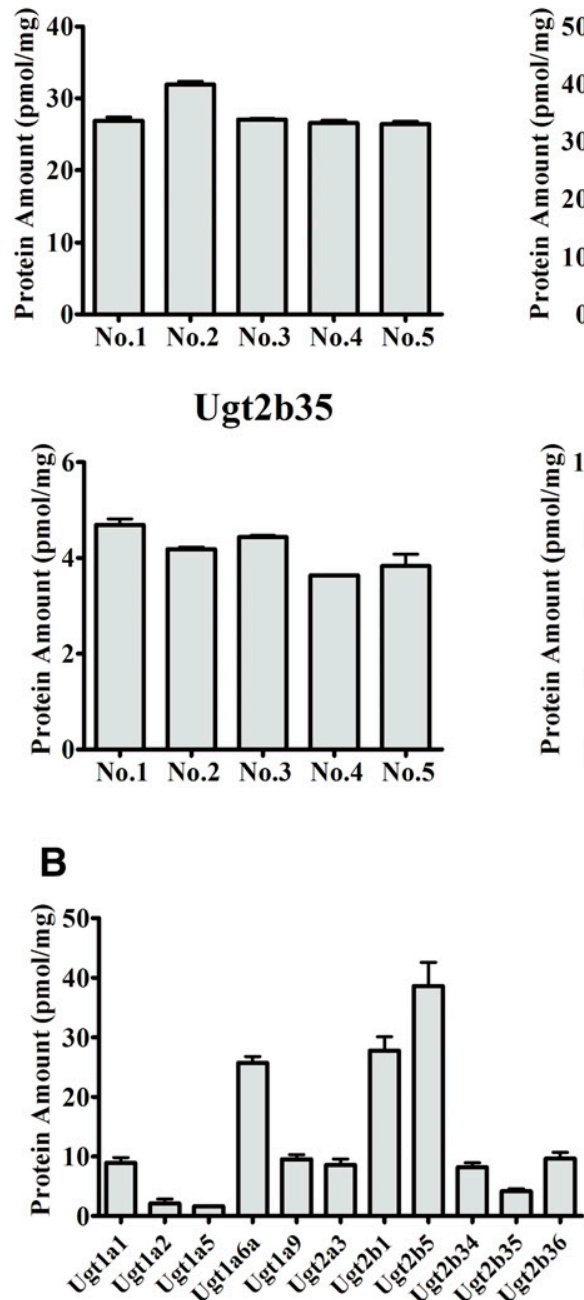

Ugt1a2

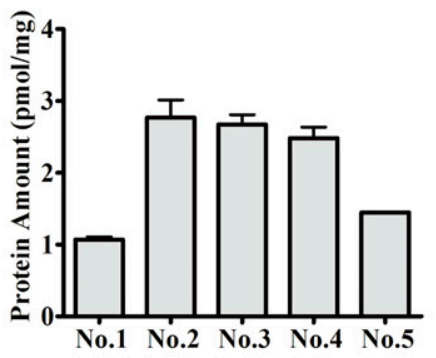

Ugt1a9

Ugt1a5
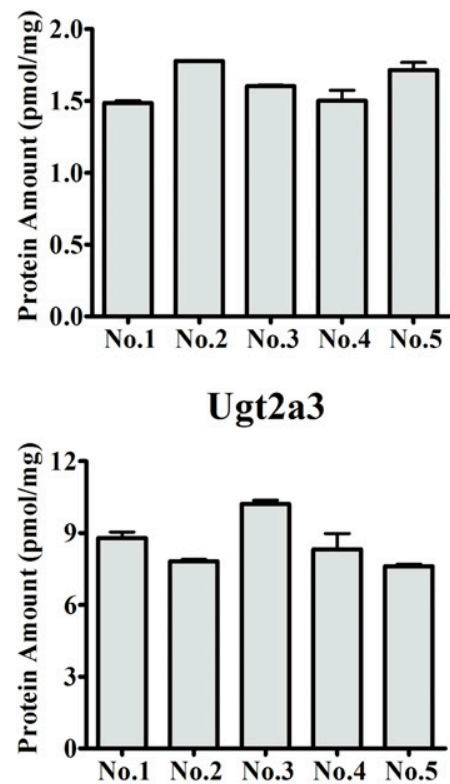

Ugt2b34

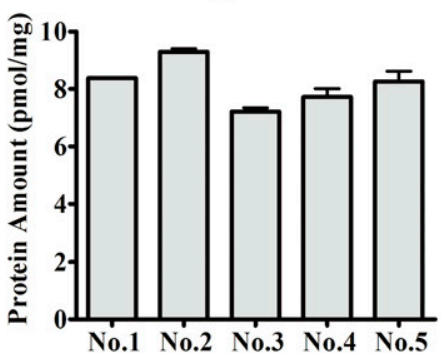

Ugt2b36

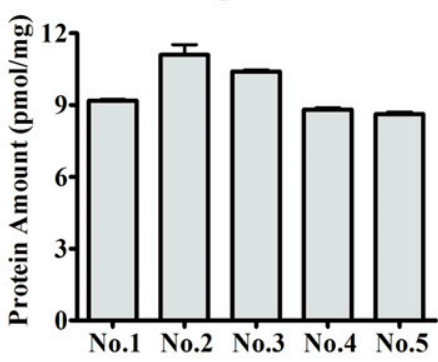

C

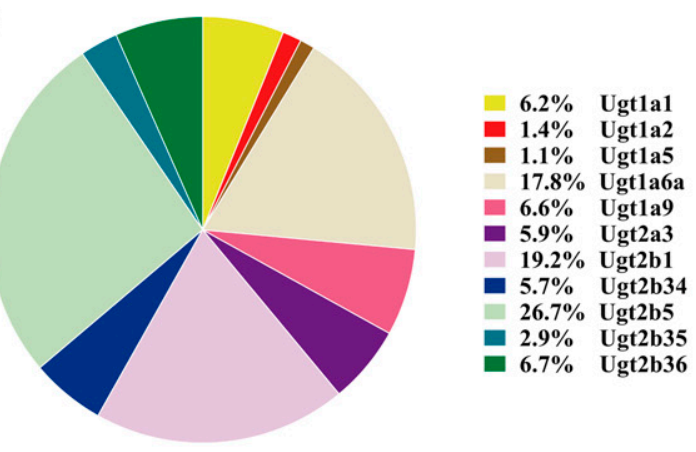

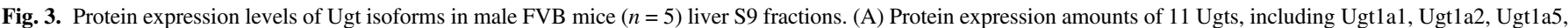

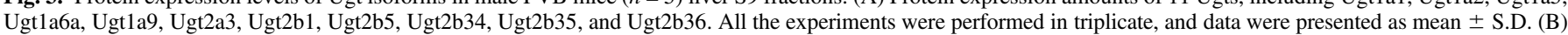

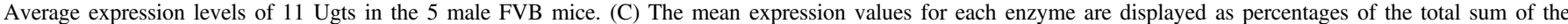
quantified 11 Ugts present in the liver. 
A

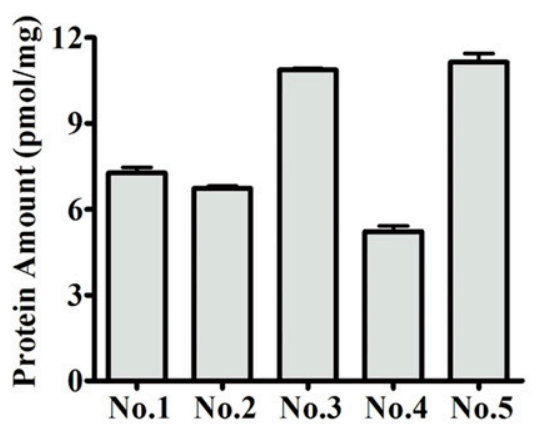

B

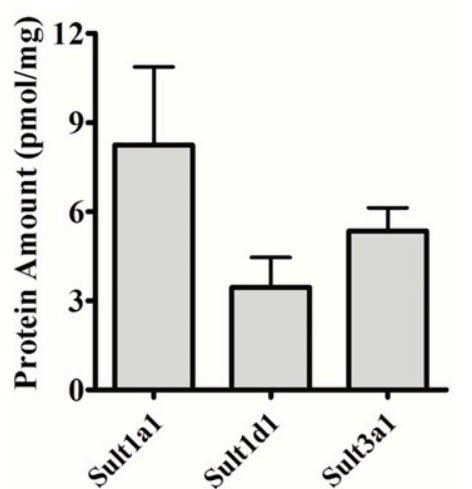

Sult1d1

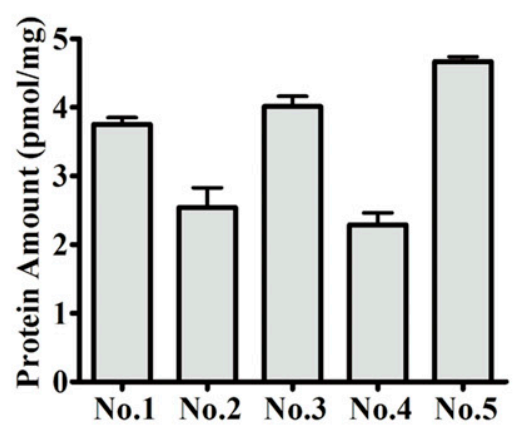

C

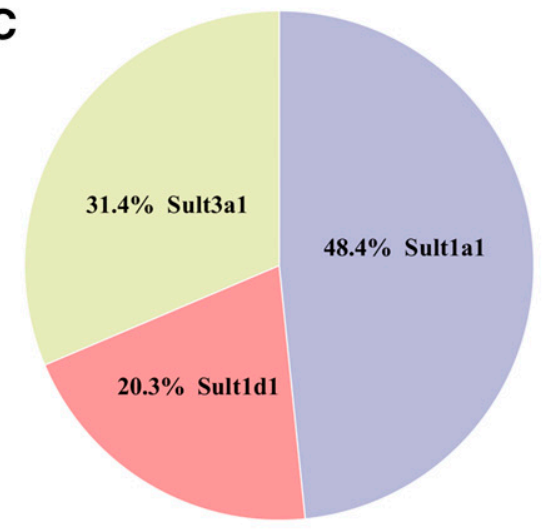

Sult3a1

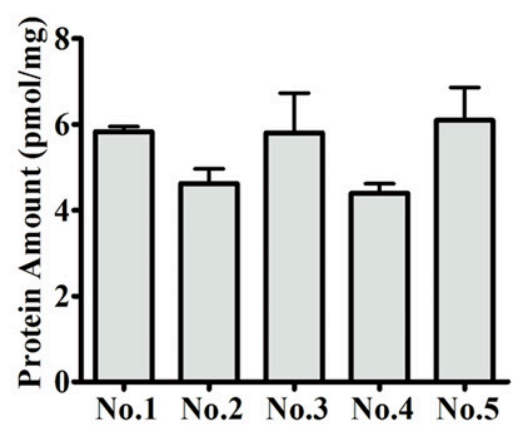

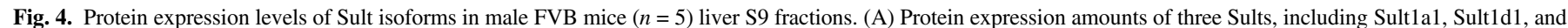

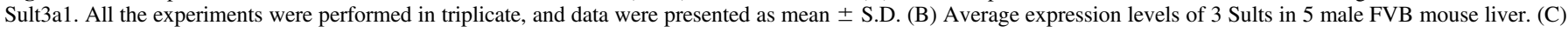
The mean expression values for each enzyme were displayed as percentages of the total sum quantified 3 Sults present in the liver.

Enzyme Expression Profiles of 24 DMEs in S9 Fractions Prepared from Female FVB Mice. As shown in Fig. 5A-7A, the protein expression levels of the individual isoforms of DMEs were also determined in five female mouse liver S9 fractions. Cyp2c29, Ugt1a6a, and Sultlal had the highest protein expression levels, at 48.7 (Fig. 5B or Supplemental Table 6), 24.4 (Fig. 6B or Supplemental Table 6), and 32.1 $\mathrm{pmol} / \mathrm{mg}$ protein (Fig. 7B or Supplemental Table 6), respectively. Overall, in female mice, Cyps, Ugts, and Sults were expressed in the following rank order, respectively: Cyp2c29 > Cyp2e1 > Cyp2c39 > Cyp2d22 > Cyp3a11 > Cyp1a2 $>$ Cyp3a25 $>$ Cyp1b1 > Cyp27a1 > Cyp7a1; Ugt1a6a $>$ Ugt2b5 > Ugt1a1 $>$ Ugt2b1 $>$ Ugt2a3 $>$ Ugt2b34 > Ugt1a9 $>$ Ugt2b36 > Ugt2b35 > Ugt1a5 > Ugt1a2; Sult1a1 > Sult3a1 > Sult1d1. In addition, when the expression levels of each Cyp, Ugt, or Sult isoforms were compared. Cyp1a2 was found to be expressed at a significant lower level than Cyp2c29 and Cyp2e1 (more than 3- or 4-fold). Meanwhile, the expression level of Cyp2c29 was significantly higher ( $>2$-fold) than those of Cyp2d22 and Cyp3a11. Ugtla6a was expressed at a significant higher level than the other isoforms (except for Ugt2b5). The protein expression level of Sult1a1 was higher than that of Sult1d1 (>3-fold).

Comparison of 24 DMEs Protein Amounts in Male and Female FVB Mice. The protein expression levels of $24 \mathrm{DME}$ isoforms in male mouse liver S9 fractions were compared against those in females. As shown in Fig. 8A, males and females displayed no difference in the protein expression levels of Cyp2e1, Cyp3a11, Cyp1b1, and Cyp3a25, whereas the protein expression level of Cyp2c39, Cyp2d22, and Cyp7a1 protein was female specific. Cyp2c39 expression was significant higher in females than in males (more than 12-fold). Three isoforms (i.e., Cyp2c29, Cyp1a2, and Cyp27a1) were male predominant. As shown in Fig. 8B, Ugtla1 and Ugt1a5 exhibited higher expression levels in females than in male mice. By contrast, Ugt2b1, Ugt2b5, and Ugt2b36 were predominantly expressed in the liver of male mice. No sex difference was noted in the protein amounts of Ugt1a2, Ugt1a6a, Ugt1a9, Ugt2a3, Ugt2b34, and Ugt2b35. In addition, female mice displayed higher protein expression levels of the measured Sult isoforms than male mice (Fig. 8C). Sult1a1 and Sult3a1 were higher in females (more than 4-fold and 7-fold, respectively) than in males. All differences were statistically significant. These data suggested that marked differences in gender-specific expression of DME isoforms exist in mice, and these differences potentially influence drug metabolism and pharmacokinetics.

\section{Discussion}

In this study, we first simultaneously determined the absolute protein expression levels of 24 metabolic enzymes in FVB mice using a UHPLC-MS/MS approach. Our results showed that 10 Cyps had detectable expression in FVB mice. As depicted in Fig. 8A, the most abundant Cyp was Cyp2c29, followed by Cyp2e1, in both male and female mice. Moreover, the isoforms differed in rank between male and female mice, as shown in Fig. 2C and Fig. 5C. The rank order of hepatic enzymes in male FVB mice was in accordance with humans. As previously reported, CYP2E1 is the most abundant CYP in the liver, accounting for $32.0 \%$ of the total content, followed by CYP2C9 (18.8\%), CYP3A4 (16.5\%), CYP2C8 (13.6\%), CYP1A2 (6.7\%), CYP2B6 (4.8\%), CYP2D6 (4.7\%), CYP2C19 (1.1\%), and CYP3A5 (1.0\%) (Gröer et al., 2014; Yan et al., 2015). In female mice, the rank order of Cyp isoforms, including Cyp2c39 and Cyp2d22, which mainly attributed to sex difference, were drastically increased (ranks 3 and 4, respectively). 
A

Cyp1a2
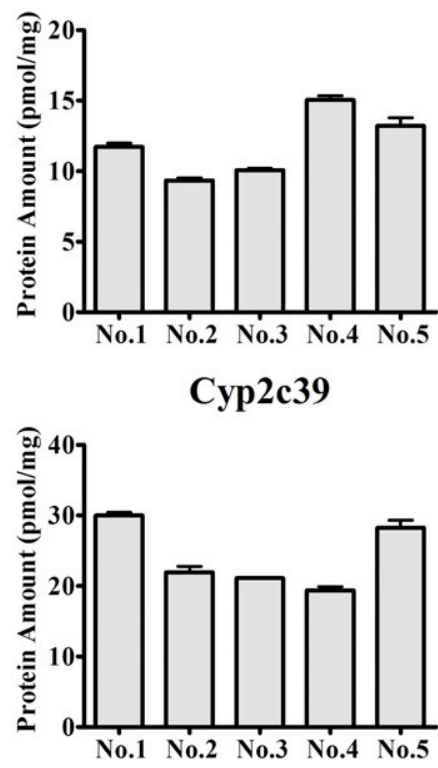

Cyp3a11

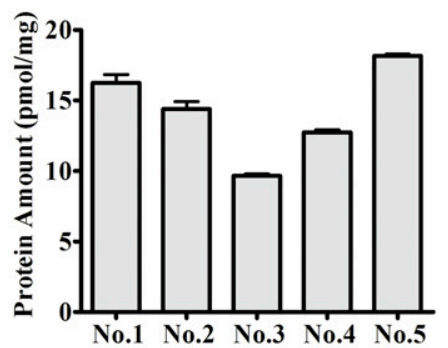

Cyp27a1

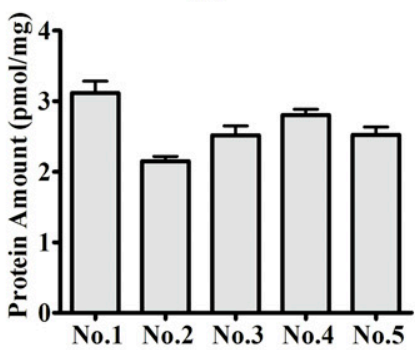

B

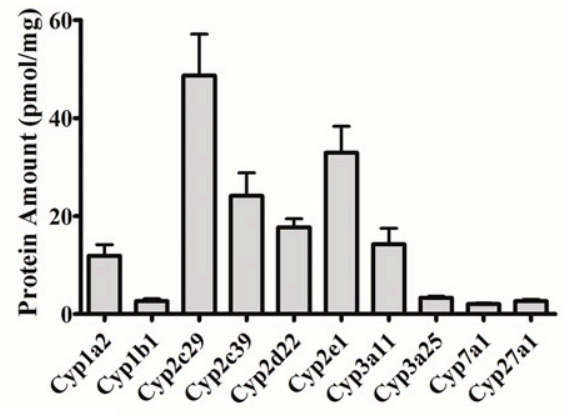

Cyp1b1
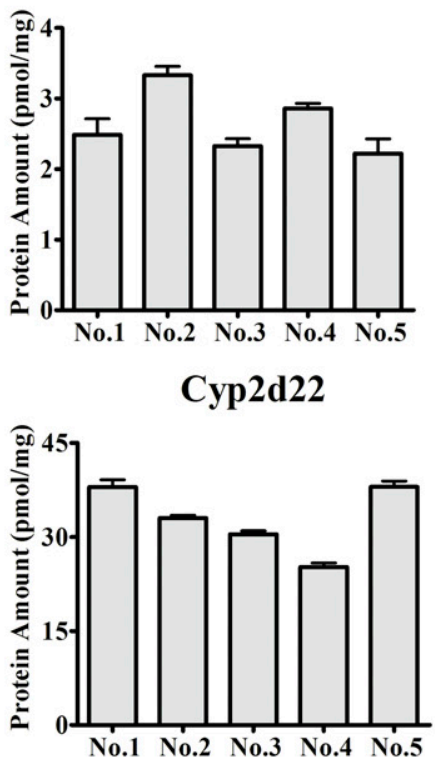

Cyp3a25

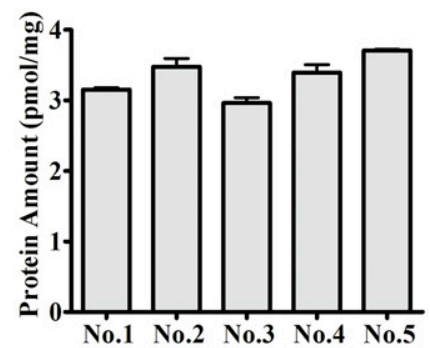

Cyp2c29
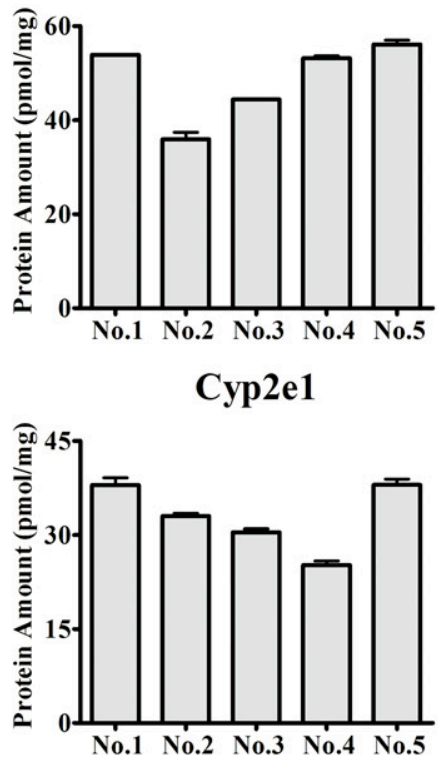

Cyp7a1

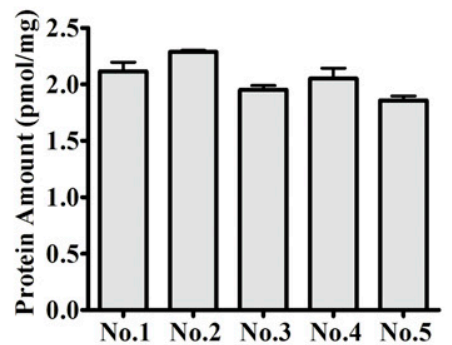

C

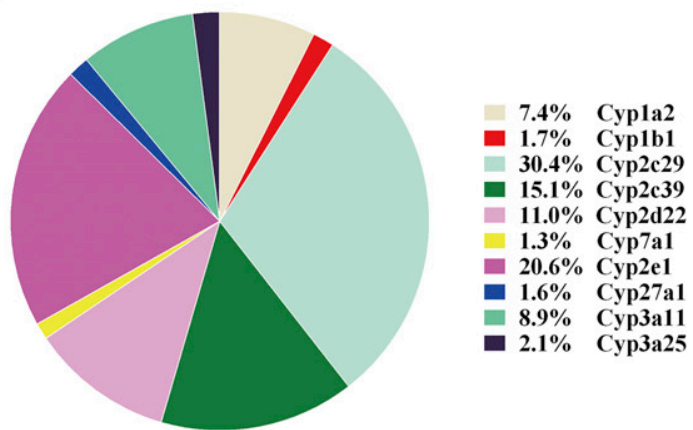

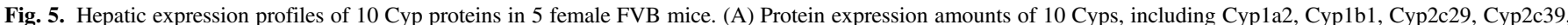

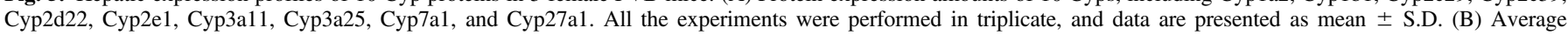
expression levels of 10 Cyps in 5 FVB mice. (C) The relative abundance of hepatic 10 Cyps based on average concentrations. 
A

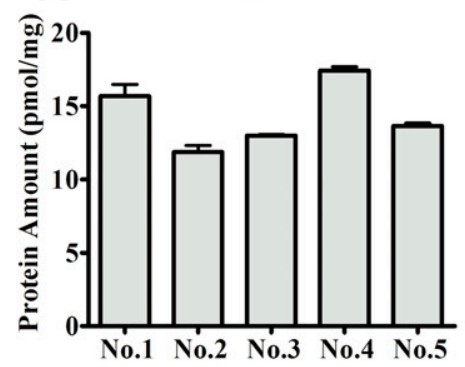

Ugt1a6a

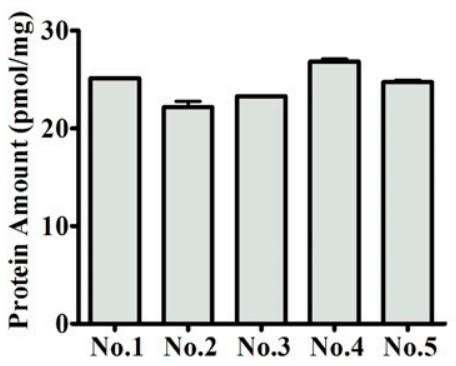

Ugt2b1

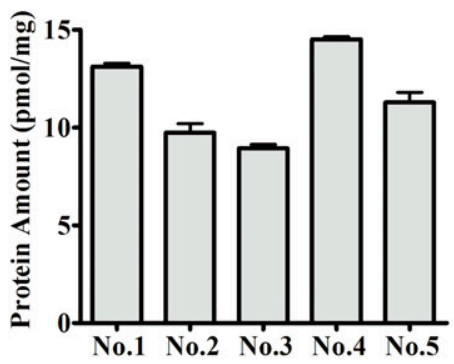

Ugt2b35

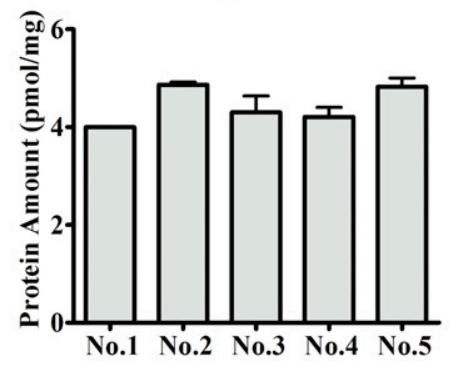

B

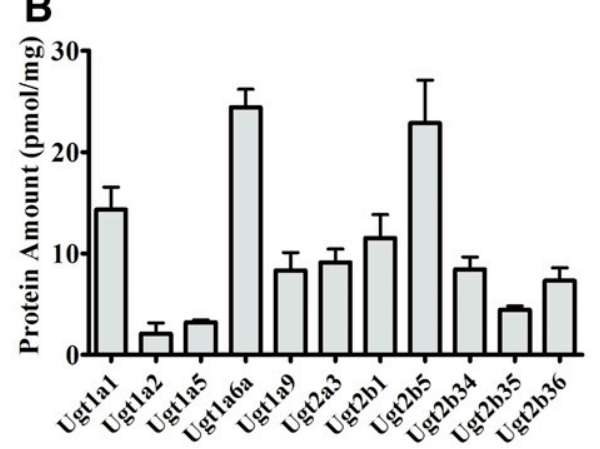

Ugt1a2

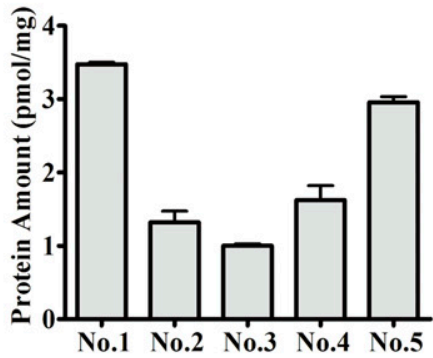

Ugt1a9

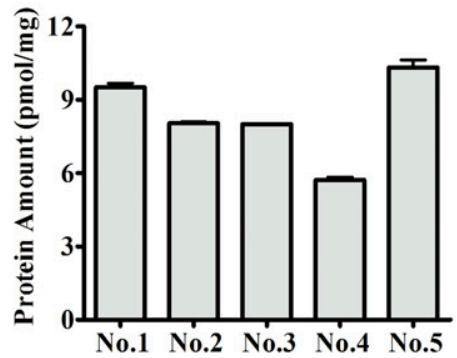

Ugt2b5

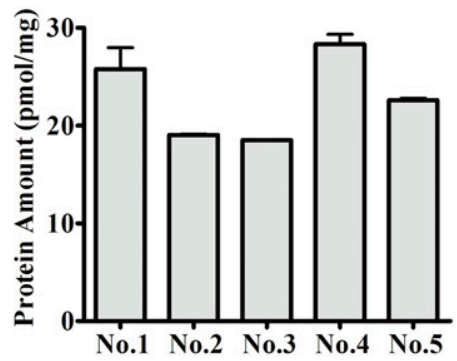

Ugt2b36

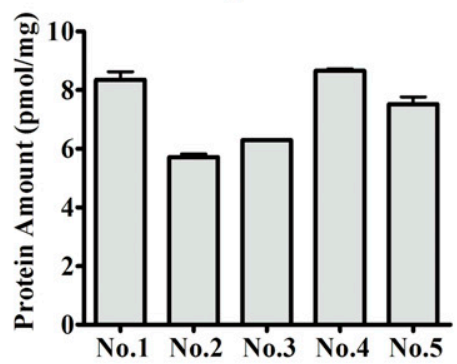

C

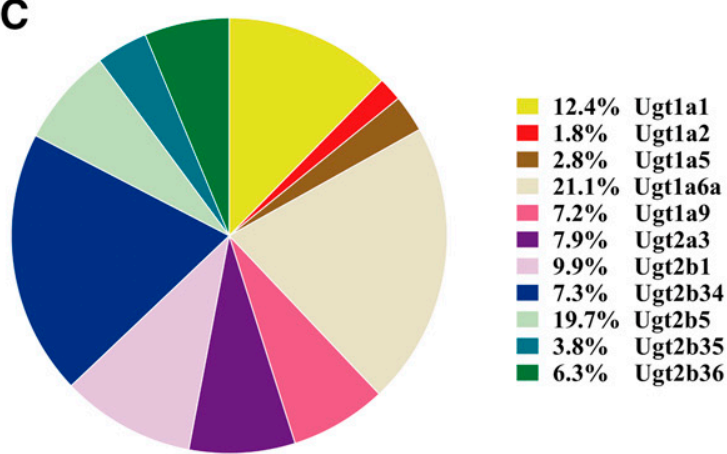

Ugt1a5
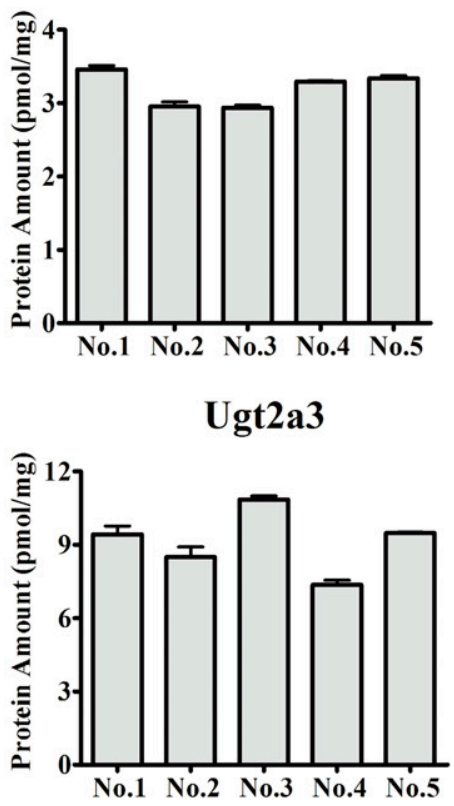

Ugt2b34

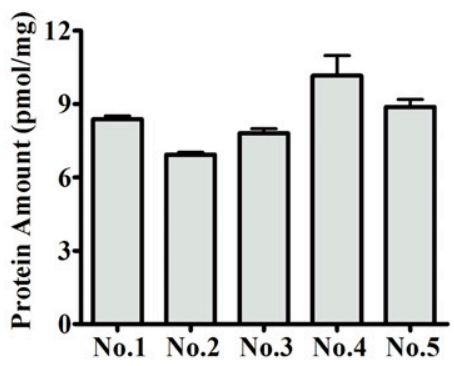

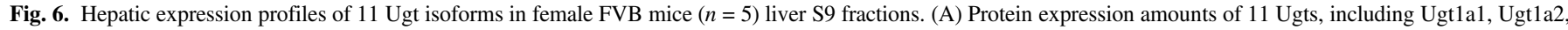

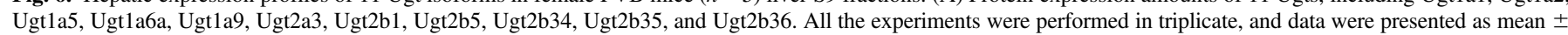
S.D. (B) Average expression levels of 11 Ugts in 5 FVB mice. (C) The relative abundance of hepatic 11 Ugts based on average concentrations. 
A

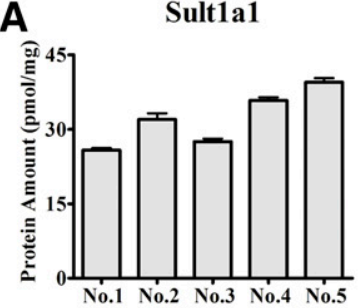

B

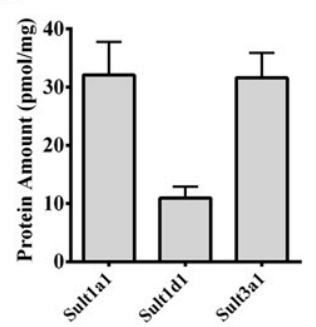

Sult1d1

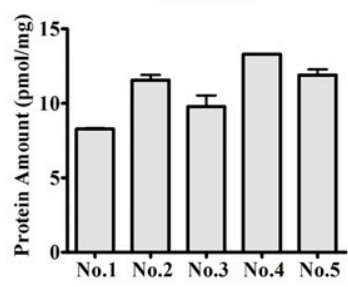

C

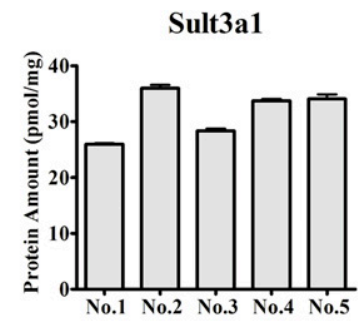

Fig. 7. Protein expression levels of DME isoforms in female FVB mice $(n=5)$ liver S9 fractions. (A) Protein expression amounts of 3 Sults, including Sult1a1, Sult1d1, and Sult3a1. All the experiments were performed in triplicate, and data were presented as mean \pm S.D. (B) Average expression levels of 3 Sults in 5 FVB mice. (C) The relative abundance of hepatic 3 Sults based on average concentrations.
In humans, it has been reported that UGT2B7 was the most abundant UGT ( $28 \%$ of the total). UGT2B4 accounted for $13 \%$ of the total protein amount. UGT2B15 and UGT1A4 each accounted for 12\% of the total UGT protein content, followed by UGT1A1 (10\%), UGT1A6 (10\%), UGT1A3 (7\%), UGT1A9 (7\%), UGT2B10 (4\%), and UGT2B17 (4\%). In mice, eight, three, and seven functional members have been identified for the Ugt1a, Ugt2a, and Ugt2b subfamilies (namely, Ugt1a1, Ugt1a2, Ugt1a5, Ugt1a6a, Ugt1a7c, Ugt1a8, Ugt1a9, Ugt1a10; Ugt2a1, Ugt2a2, Ugt2a3; and Ugt2b1, Ugt2b5, Ugt2b34, Ugt2b35, Ugt2b36, Ugt2b37, and Ugt2b38), respectively (Rowland et al., 2013; Oda et al., 2015). As shown in Fig. 3C, the protein amount of Ugts in male FVB mice consisted of Ugt2b5 (26\%), Ugt2b1 (19\%), Ugt1a6a (18\%), Ugt2b36 (7\%), Ugt1a1, Ugt1a9, Ugt2a3, Ugt2b34 (6\% of total, respectively), Ugt $2 \mathrm{~b} 35$ (3\% of total), and Ugt1a2 (1\% of total). Interestingly, Ugtla1 exhibited significant higher expression in female than in male mice $(\sim 2$ fold). It is well known that human UGT1A1 plays a vital role in the glucuronidation of bilirubin, which leads to hyperbilirubinemia. Therefore, our results on expression level of Ugtla1 in FVB mice may predict the metabolism of bilirubin in human.

In humans, the data showed SULT1A1, SULT2A1, SULT1B1, and SULT1E were detected in human liver, accounting for 53\%, 27\%, 14\%, and $6 \%$ of five Sults isoforms, respectively. However, SULT1A3 was not detectable (Riches et al., 2009). Alnouti and Klaassen (2006) conducted a branched DNA signal amplification assay and found that Sult1a1, Sult1c1, Sult1c2, Sult1d1, Sult2a1, and Sult3a1 were expressed in mouse liver. However, we only detected the expression of Sult1a1, Sult1d1, and Sult3a1 at the protein level in mouse liver.

Similar to the findings of other studies that analyzed the mRNA level and activity of DMEs, the protein expression of several DMEs showed sex specificity. For example, Cyp1a2 and Cyp2c29 exhibited higher expression in males, whereas Cyp2d22 and Cyp2c39 exhibited higher expression in females. No sex difference was observed for Cyp3a11 and Cyp2e1 in mice. A previous study showed CYP1A2 activity is slightly lower in women than in men (Hrycay and Bandiera, 2009). Benzene and chlorzoxazone, which are the substrates of CYP2E1, were found to be eliminated more slowly in women than in men in an experimental study on human exposures. Erythromycin, midazolam, verapamil, which are substrates of CYP3A4 considered as the human ortholog of mouse Cyp3a11, had higher clearance in women (Franconi et al., 2007; Hrycay and Bandiera, 2009). Cyp3a25 is a female-specific member of the CYP3A gene subfamily expressed in the mouse liver (Sakuma et al., 2000).
Cyp2d22 has been posited to be the mouse ortholog of human CYP2D6 (Blume et al., 2000). Faster clearance of dextromethorphan, sertraline, desipramine, and metoprolol has been observed in men than in women, suggesting that CYP2D6 activity may be lower in women (Franconi et al., 2007). It has been recognized that mouse Cyp2c37, Сyp2c38, Сур2c39, and Cyp2c40 are all female specific (Löfgren et al., 2008).

The published study carried out by other groups revealed that Ugt1a1 and Ugt1a5 displayed a female predominant mRNA expression. By contrast, Ugt $2 \mathrm{~b} 1$ exhibited male-predominant mRNA expression. Our results also demonstrated the female predominance of Ugt1a1 and Ugt1a5 protein expression. In comparison, Ugt2b1 and Ugt2b5 exhibited significantly higher protein amounts in male than in female mice. In general, members of the UGT1A gene family are largely conserved among species. Hence, special attention may be put on the sex differences of Ugtla family.

Sulfotransferases belong to the major phase II DMEs that sulfoconjugate various substance (e.g., biogenic amines, steroid hormones, bile acids, and drugs). Previous studies indicated that the mRNA expression of Sult1a1, Sult1d1, Sult2a1/a2, and Sult3a1 in the liver and Sult4a1 in the brain displayed sex differences, with higher mRNA levels in females compared with males (Alnouti and Klaassen, 2006). Hence, we developed a method for quantifying the protein expression of Sult1 (Sult1a1, Sult1b1, and Sult1d1), Sult3 (Sult3a1), and Sult4 (Sult4a1) superfamilies. However, it has been identified that Sult1b1 and Sult4a1 were mainly distributed in the digestive track and brain, respectively (Alnouti and Klaassen, 2006; Butcher et al., 2010). Consistently, Sult1a1, Sult1d1, and Sult3a1 were also detectable in the liver in current work, with higher protein expression in females than in males.

Immunological methods have been extensively applied for quantifying the amounts of DMEs. Although data on gene expression can indicate the presence of DMEs, these data cannot be applied to PBPK modeling because of the poor correlation between mRNA and protein levels. Meanwhile, protein quantification by WB is semiquantitative. To date, MS-based quantification has become main approach for qualitative and quantitative assays of protein. In the present study, the developed method could be used to quantify multiple enzymes with good linear range, stability, and matrix effect. Although the inaccuracy values of some isoforms were somewhat higher due to the complex sample preparation and analytical technique, our results were comparable to those of previous studies. In addition, the similar rank order and the sex 
A

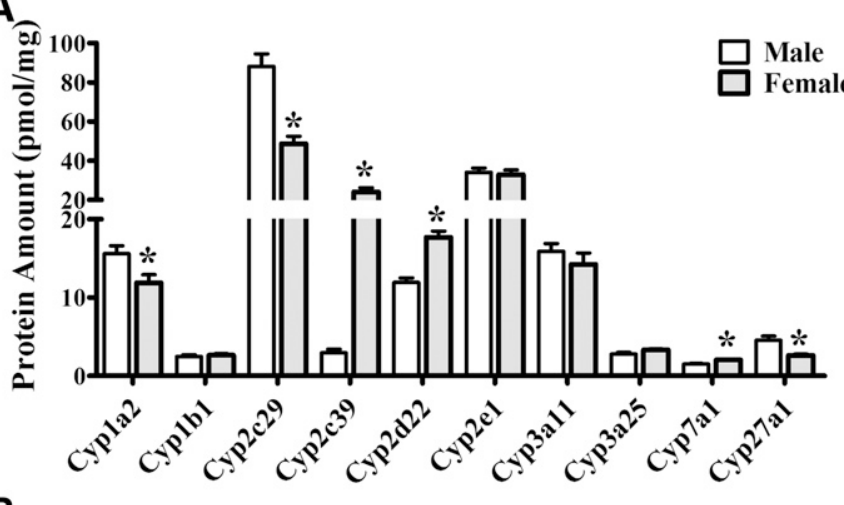

B

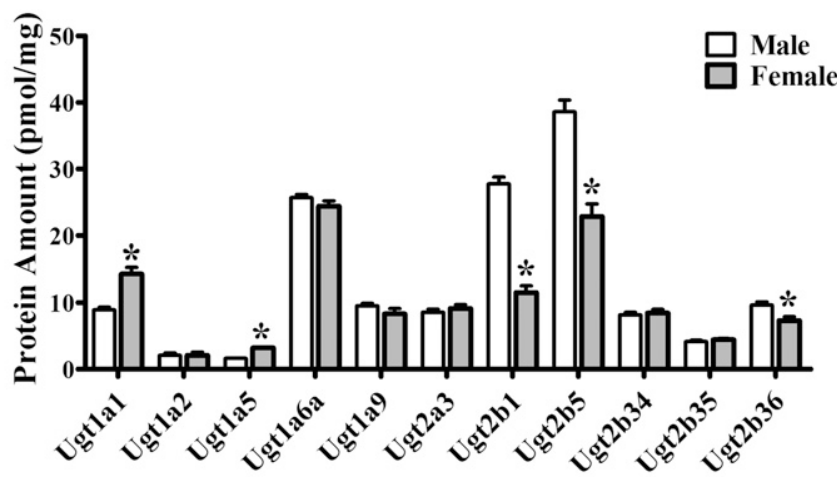

C

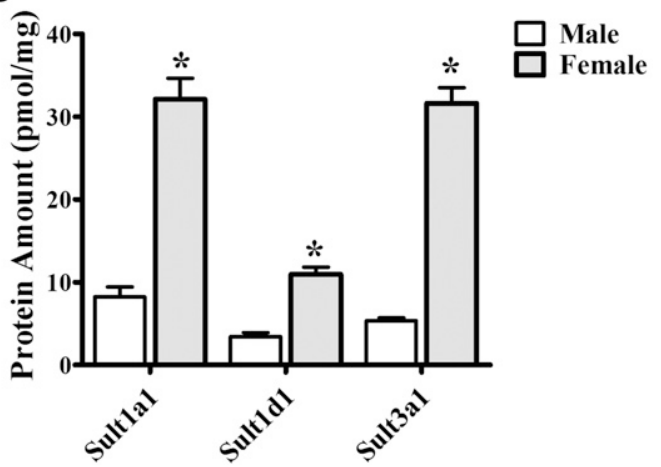

Fig. 8. Protein expression levels of DME isoforms in the liver S9 fractions of male FVB mice compared with female FVB mice ( $n=5$, respectively). (A) Protein expression amounts of 10 Cyps in male FVB mice compared with female FVB mice, including Cyp1a2, Cyp1b1, Cyp2c29, Cyp2c39, Cyp2d22, Cyp2e1, Сyp3a11, Cyp3a25, Cyp7a1, and Cyp27a1. (B) Protein expression amounts of 11 Ugts, including Ugt1a1, Ugt1a2, Ugt1a5, Ugt1a6, Ugt1a9, Ugt2a3, Ugt2b1, Ugt2b5, Ugt2b34, Ugt2b35, and Ugt2b36 in male FVB mice compared with female FVB mice. (C) Protein expression amounts of 3 Sults in male FVB mice compared with female FVB mice, including Sult1a1, Sult1d1, and Sult3a1. Data were presented as mean \pm S.D. Data analysis was performed by the way of independent sample $t$ test. *Statistical significance $(P<0.05)$.

differences of DMEs in protein levels with mRNA and activity levels suggested that the approach was accurate and precise.

The present study is the first to employ an isotope label-free UHPLCMS/MS approach to systematically and comprehensively perform studies for profiling 24 DMEs in FVB mice. Our findings suggested that the FVB animal model can be well applied for the metabolism and subsequent pharmacokinetics of substrates of these 24 enzymes.

\section{Acknowledgments}

The authors thank Zhongqiu Liu for helpful guide of the experiment and manuscript and Lijun Zhu for assistance with preparation the manuscript.

\section{Authorship Contributions}

Participated in research design: Chen, Yan, and Liu.

Conducted experiments: Chen, $\mathrm{Li}$, Zheng, Xie, and Zeng.

Contributed new reagents or analytic tools: Zhu, Lu, Qi, Wang, $\mathrm{Hu}$, and Liu. Performed data analysis: Chen, Zhu, Li, Zheng, Yu, and Jiang.

Wrote or contributed to the writing of the manuscript: Chen, Zhu, and Liu.

\section{References}

Achour B, Russell MR, Barber J, and Rostami-Hodjegan A (2014) Simultaneous quantification of the abundance of several cytochrome P450 and uridine 5'-diphospho-glucuronosyltransferase enzymes in human liver microsomes using multiplexed targeted proteomics. Drug Metab Dispos 42:500-510.

Agarwal S, Uchida Y, Mittapalli RK, Sane R, Terasaki T, and Elmquist WF (2012) Quantitative proteomics of transporter expression in brain capillary endothelial cells isolated from P-glycoprotein (P-gp), breast cancer resistance protein (Bcrp), and P-gp/Bcrp knockout mice. Drug Metab Dispos 40:1164-1169.

Alnouti Y and Klaassen CD (2006) Tissue distribution and ontogeny of sulfotransferase enzymes in mice. Toxicol Sci 93:242-255.

Blume N, Leonard J, Xu ZJ, Watanabe O, Remotti H, and Fishman J (2000) Characterization of Cyp2d22, a novel cytochrome P450 expressed in mouse mammary cells. Arch Biochem Biophys 381:191-204.

Butcher NJ, Mitchell DJ, Burow R, and Minchin RF (2010) Regulation of mouse brain-selective sulfotransferase sult4a1 by cAMP response element-binding protein and activating transcription factor-2. Mol Pharmacol 78:503-510.

Fallon JK, Neubert H, Goosen TC, and Smith PC (2013a) Targeted precise quantification of 12 human recombinant uridine-diphosphate glucuronosyl transferase $1 \mathrm{~A}$ and $2 \mathrm{~B}$ isoforms using nano-ultra-high-performance liquid chromatography/tandem mass spectrometry with selected reaction monitoring. Drug Metab Dispos 41:2076-2080.

Fallon JK, Neubert H, Hyland R, Goosen TC, and Smith PC (2013b) Targeted quantitative proteomics for the analysis of 14 UGT1As and -2Bs in human liver using NanoUPLC-MS/MS with selected reaction monitoring. J Proteome Res 12:4402-4413.

Franconi F, Brunelleschi S, Steardo L, and Cuomo V (2007) Gender differences in drug responses. Pharmacol Res 55:81-95.

Ge S, Gao S, Yin T, and Hu M (2015) Determination of pharmacokinetics of chrysin and its conjugates in wild-type FVB and Bcrp1 knockout mice using a validated LC-MS/MS method. J Agric Food Chem 63:2902-2910.

Girard SD, Escoffier G, Khrestchatisky M, and Roman FS (2016) The FVB/N mice: A well suited strain to study learning and memory processes using olfactory cues. Behav Brain Res 296: 254-259.

Goelz MF, Mahler J, Harry J, Myers P, Clark J, Thigpen JE, and Forsythe DB (1998) Neuropathologic findings associated with seizures in FVB mice. Lab Anim Sci 48:34-37.

Gröer C, Busch D, Patrzyk M, Beyer K, Busemann A, Heidecke CD, Drozdzik M, Siegmund W, and Oswald S (2014) Absolute protein quantification of clinically relevant cytochrome P450 enzymes and UDP-glucuronosyltransferases by mass spectrometry-based targeted proteomics. $J$ Pharm Biomed Anal 100:393-401.

Hrycay EG and Bandiera SM (2009) Expression, function and regulation of mouse cytochrome P450 enzymes: comparison with human P450 enzymes. Curr Drug Metab 10:1151-1183.

Joo J, Lee B, Lee T, and Liu KH (2014) Screening of six UGT enzyme activities in human liver microsomes using liquid chromatography/triple quadrupole mass spectrometry. Rapid Commun Mass Spectrom 28:2405-2414.

Löfgren S, Baldwin RM, Hiratsuka M, Lindqvist A, Carlberg A, Sim SC, Schülke M, Snait M, Edenro A, Fransson-Steen R, et al. (2008) Generation of mice transgenic for human CYP2C18 and CYP2C19: characterization of the sexually dimorphic gene and enzyme expression. Drug Metab Dispos 36:955-962.

Longo UG, Forriol F, Campi S, Maffulli N, and Denaro V (2011) Animal models for translational research on shoulder pathologies: from bench to bedside. Sports Med Arthrosc Rev 19:184-193.

Margaillan G, Rouleau M, Klein K, Fallon JK, Caron P, Villeneuve L, Smith PC, Zanger UM, and Guillemette C (2015) Multiplexed targeted quantitative proteomics predicts hepatic glucuronidation potential. Drug Metab Dispos 43:1331-1335.

Oda S, Fukami T, Yokoi T, and Nakajima M (2015) A comprehensive review of UDPglucuronosyltransferase and esterases for drug development. Drug Metab Pharmacokinet 30: $30-51$.

Ohno S and Nakajin S (2009) Determination of mRNA expression of human UDPglucuronosyltransferases and application for localization in various human tissues by real-time reverse transcriptase-polymerase chain reaction. Drug Metab Dispos 37:32-40.

Riches Z, Stanley EL, Bloomer JC, and Coughtrie MW (2009) Quantitative evaluation of the expression and activity of five major sulfotransferases (SULTs) in human tissues: the SULT "pie". Drug Metab Dispos 37:2255-2261.

Rowland A, Miners JO, and Mackenzie PI (2013) The UDP-glucuronosyltransferases: their role in drug metabolism and detoxification. Int J Biochem Cell Biol 45:1121-1132.

Sakamoto A, Matsumaru T, Ishiguro N, Schaefer O, Ohtsuki S, Inoue T, Kawakami H, and Terasaki T (2011) Reliability and robustness of simultaneous absolute quantification of drug transporters, cytochrome P450 enzymes, and Udp-glucuronosyltransferases in human liver tissue by multiplexed MRM/selected reaction monitoring mode tandem mass spectrometry with nanoliquid chromatography. J Pharm Sci 100:4037-4043.

Sakuma T, Takai M, Endo Y, Kuroiwa M, Ohara A, Jarukamjorn K, Honma R, and Nemoto N (2000) A novel female-specific member of the CYP3A gene subfamily in the mouse liver. Arch Biochem Biophys 377:153-162.

Schaefer O, Ohtsuki S, Kawakami H, Inoue T, Liehner S, Saito A, Sakamoto A, Ishiguro N, Matsumaru T, Terasaki T, et al. (2012) Absolute quantification and differential expression of drug transporters, cytochrome P450 enzymes, and UDP-glucuronosyltransferases in cultured primary human hepatocytes. Drug Metab Dispos 40:93-103.

Seibert C, Davidson BR, Fuller BJ, Patterson LH, Griffiths WJ, and Wang Y (2009) Multipleapproaches to the identification and quantification of cytochromes P450 in human liver tissue by mass spectrometry. J Proteome Res 8:1672-1681. 
Shi R, Ma B, Wu J, Wang T, and Ma Y (2015) Rapid and accurate liquid chromatography and tandem mass spectrometry method for the simultaneous quantification of ten metabolic reactions catalyzed by hepatic cytochrome P450 enzymes. J Sep Sci 38:3363-3373.

Sridar C, Hanna I, and Hollenberg PF (2013) Quantitation of UGT1A1 in human liver microsomes using stable isotope-labelled peptides and mass spectrometry based proteomic approaches Xenobiotica 43:336-345.

Tang L, Feng Q, Zhao J, Dong L, Liu W, Yang C, and Liu Z (2012) Involvement of UDPglucuronosyltranferases and sulfotransferases in the liver and intestinal first-pass metabolism of seven flavones in C57 mice and humans in vitro. Food Chem Toxicol 50: $1460-1467$.

Yan T, Gao S, Peng X, Shi J, Xie C, Li Q, Lu L, Wang Y, Zhou F, Liu Z, et al. (2015) Significantly decreased and more variable expression of major CYPs and UGTs in liver microsomes prepared from HBV-positive human hepatocellular carcinoma and matched pericarcinomatous tissues determined using an isotope label-free UPLC-MS/MS method. Pharm Res 32:1141-1157.
Zaher H, Khan AA, Palandra J, Brayman TG, Yu L, and Ware JA (2006) Breast cancer resistance protein (Bcrp/abcg2) is a major determinant of sulfasalazine absorption and elimination in the mouse. Mol Pharm 3:55-61.

Zhang X, Dong D, Wang H, Ma Z, Wang Y, and Wu B (2015) Stable knock-down of efflux transporters leads to reduced glucuronidation in UGT1A1-overexpressing HeLa cells: the evidence for glucuronidation-transport interplay. Mol Pharm 12:1268-1278.

Zhu W, Xu H, Wang SW, and Hu M (2010) Breast cancer resistance protein (BCRP) and sulfotransferases contribute significantly to the disposition of genistein in mouse intestine. AAPS J 12:525-536.

Address correspondence to: Prof. Zhongqiu Liu, International Institute for Translational Chinese Medicine, Guangzhou University of Chinese Medicine, Guangzhou, Guangdong, 510006, PR China. E-mail: liuzq@gzucm.edu.cn 